\title{
A Combinatorial Property of Convex Sets
}

\author{
M. Abellanas, ${ }^{1}$ G. Hernandez, ${ }^{1}$ R. Klein, ${ }^{2}$ V. Neumann-Lara, ${ }^{3}$ and J. Urrutia ${ }^{4}$ \\ ${ }^{1}$ Facultad de Informática, Dept. de Matemática Aplicada, \\ Universidad Politecnica de Madrid, Campus de Montegancedo, $s / n$, \\ Boadilla del Monte, 28660 Madrid, Spain \\ \{mavellanas,gregorio\}@fi.upm.es \\ ${ }^{2}$ Praktische Informatik VI, Fern Universität Hagen, \\ Elberfelder Strasse 95, 5800 Hagen, Germany \\ Rolf.Klein@FernUni-Hagen.de \\ ${ }^{3}$ Universidad Nacional Antonoma de México, Mexico \\ ${ }^{4}$ Department of Computer Science, University of Ottawa, \\ Ottawa, Ontario, Canada K1N 6N5 \\ jorge@csi.uottawa.ca
}

\begin{abstract}
A known result in combinatorial geometry states that any collection $P_{n}$ of points on the plane contains two such that any circle containing them contains $n / c$ elements of $P_{n}, c$ a constant. We prove: Let $\Phi$ be a family of $n$ noncrossing compact convex sets on the plane, and let $S$ be a strictly convex compact set. Then there are two elements $S_{l}, S$, of $\Phi$ such that any set $S^{\prime}$ homothetic to $S$ that contains them contains $n / c$ elements of $\Phi, c$ a constant $\left(S^{\prime}\right.$ is homothetic to $S$ if $S^{\prime}=\lambda S+\mathbf{v}$, where $\lambda$ is a real number greater than 0 and $\mathbf{v}$ is a vector of $\mathfrak{\Re}^{2}$ ). Our proof method is based on a new type of Voronoi diagram, called the "closest covered set diagram" based on a convex distance function. We also prove that our result does not generalize to higher dimensions; we construct a set $\Phi$ of $n$ disjoint convex sets in $\mathfrak{R}^{3}$ such that for any nonempty subset $\Phi_{H}$ of $\Phi$ there is a sphere $S_{H}$ containing all the elements of $\Phi_{H}$, and no other element of $\Phi$.
\end{abstract}

\section{Introduction}

A known result in combinatorial geometry asserts that any collection $P_{n}$ of $n$ points on the plane contains two elements $u, v \in P_{n}$ such that any circle containing them contains at least $[n / c\rceil$ points of $P_{n}$. The first proved value for $c$ was $60[10]$, which was successively improved to 30 [2], then to $\left\lceil\frac{84}{5}\right\rceil[6]$ and, at this point, the best known value for $c$ is 4.7 (see [4]). Containment problems between families of points and circles 
originated from the study of circle orders, i.e., partial orders obtained from containment relations of families of circles on the plane; see [5] and [11].

Two compact convex sets $S_{i}$ and $S_{j}$ cross each other if $S_{i}-S_{j}$ and $S_{j}-S_{\imath}$ are not connected. A collection $\Phi=\left\{S_{1}, \ldots, S_{n}\right\}$ of compact convex sets is called noncrossing if no elements of it cross. In this paper we prove the following: Let $S$ be a strictly compact convex set (i.e., it is closed, bounded, and has no piecewise linear segments on its boundary) and let $\Phi=\left\{S_{1}, \ldots, S_{n}\right\}$ be a family of noncrossing compact convex sets. Then there are two elements $S_{i}, S_{j} \in \Phi$ such that any set $S^{\prime}$ homothetic to $S$ containing them contains at least $\lceil(n-2) / 30\rceil$ elements of $\Phi$. ( $S^{\prime}$ is homothetic to $S$ if $S^{\prime}=\lambda S+\mathbf{v}$, where $\lambda$ is a real value greater than 0 and $\mathbf{v}$ is a vector of $\Re^{2}$ ). Our proof relies on a new type of Voronoi diagram, which we suggest calling the "closest covered set Voronoi diagram." In general, a Voronoi diagram is a partitioning of the plane according to a distance function defined between the points of the plane and a collection of sites; in our case the elements of $\Phi$. We study the case in which the sites form a family of noncrossing compact convex sets in the plane, and where each point $x$ of the plane belongs to the first site contained in a homothetically expanding compact and convex oval "centered" at $x$. The circle need not be Euclidean; we can take any strictly convex set $S$ containing $x$ in its interior, and consider growing homothetic copies of $S$. In other words, our diagram is based on the following distance function: With each site $S_{i}$ and any point $x$, the distance from $x$ to $S_{i}$ is the distance from $x$ to the farthest point of $S_{i}$ under a fixed convex distance function.

The original result for points and circles proved in [10] has been generalized to higher dimensions in [2]; in [1] a surprising generalization using collections of points and ellipsoids in Euclidean spaces was given. Our new result seems to be of a different nature than previous ones for it does not generalize to higher dimensions. We present a family $\Psi$ of $n$ disjoint convex sets in $\Re^{3}$ with the property that for every subset $\Psi_{H}$ of $\Psi$ here is a sphere that contains all the elements of $\Psi_{H}$ and no other element of $\Psi$.

\section{Main Results}

\subsection{Dimension 3}

We start our paper by showing that the main result of this paper does not generalize to higher dimensions.

To this end, consider the set $\Sigma=\left\{P_{1}, \ldots, P_{n}\right\}$ of $n$ polygons defined as follows: Let $C$ be a circle on the plane $z=0$ of $\mathfrak{R}^{3}$ together with a set of $2^{n}-1$ disjoint intervals on it labeled $C_{H}$, where $H$ runs over all nonempty subsets of $I_{n}=\{1, \ldots, n\}$. For every $H$ let $C_{H}^{-}$and $C_{H}^{+}$be the initial and final points of $C_{H}$ in the counterclockwise direction. For every nonempty subset $H=\{i(1), \ldots, i(k)\}$ of $I_{n}$ take $n$ equidistant points in the interior of $C_{H}$ and label the first $k$ of them in the counterclockwise direction with the integers $i(1), \ldots, i(k)$ and the remaining $n-k$ points with the integers in $I_{n}-\{i(1), \ldots, i(k)\}$ (see Fig. 1). Let $\alpha_{H}$ be any point in $C_{H}$ to the right of $i(k)$ appearing before any of the points on $C_{H}$ with a label in $I_{n}-\{i(1), \ldots, i(k)\}$. For every $i \in I_{n}$ let $P_{i}$ be the convex closure of all of the $2^{n}-1$ points on $C$ labeled $i$.

It is easy to see that, for every $H$, there is a circle that intersects $C$ at $C_{H}^{+}$and $\alpha_{H}$, 


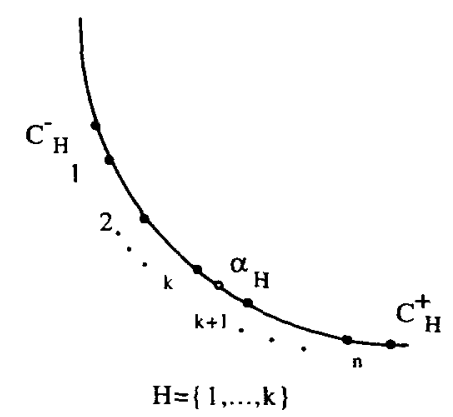

Fig. 1

containing only those elements of $\Sigma$ in $\Sigma_{H}=\left\{P_{l}, i \in H\right\}$. For every $i$, let $P_{i}^{\prime}$ be a translation of $P_{i}$ in the direction of the $z$-axis by a small $\varepsilon_{i}, i=1, \ldots, n ; \varepsilon_{i} \neq \varepsilon_{\jmath}$, $i \neq j$. Let $\Gamma=\left\{P_{1}^{\prime}, \ldots, P_{n}^{\prime}\right\}$. For every subset $H=\{i(1), \ldots, i(k)\}$ of $I_{n}$ let $\Gamma_{H}=$ $\left\{P_{t(1)}, \ldots, P_{i(k)}^{\prime}\right\}$. It is now easy to see that for any such $H$ there is a sphere in $\mathfrak{R}^{3}$ that contains only those elements of $\Gamma$ in $\Gamma_{H}$.

Thus we have proved:

Theorem 1. For every $n \geq 1$ there exists a family $\Gamma$ of $n$ disjoint convex sets in $\mathfrak{R}^{3}$ such that for any subset $\Gamma_{H}$ of $\Gamma$ there is a sphere containing only those elements of $\Gamma$ in $\Gamma_{H}$.

Corollary 1. For any $n \geq 1$ there is a family $\Sigma=\left\{P_{1}, \ldots, P_{n}\right\}$ of $n$ mutually crossing polygons on the plane such that for every subset $\Sigma_{H} \subset \Sigma$ there is a circle containing the elements of $\Sigma_{H}$, and no other element of $\Sigma$.

\subsection{The Main Result}

Our objective in this section is to prove:

Theorem 2. Let $\Phi=\left\{S_{1}, \ldots, S_{n}\right\}$ be any family of noncrossing compact convex sets on the plane, and let $S$ be any strictly convex compact set. Then there are two elements $S_{i}, S_{j} \in \Phi$ such that any convex set $S^{\prime}$ homothetic to $S$ containing them contains at least $\lceil(n-2) / 30\rceil$ elements of $\Phi$.

Our proof is based on the following theorem, which is interesting in its own right. The proof this theorem is postponed to the next section:

Theorem 3. Let $H$ be any family of five noncrossing compact convex sets, and let $S$ be a strictly convex compact convex set. Then there are two elements $S_{i}, S_{j} \in H$ such that any $S^{\prime}$ homothetic to $S$ containing them contains another element of $H$.

We now prove Theorem 2 . 
Proof of Theorem 2. Construct a bipartite graph $G$ with $V(G)=X \cup Y$ where $X$ consists of all subsets of two of elements of $\Phi$ and $Y$ contains all of the subsets of $\Phi$ with exactly five elements. A vertex $T \in X$ is adjacent to a vertex $T^{\prime} \in Y$ iff $T \subset T^{\prime}$ and any homothetic copy of $S$ containing the elements of $T$ contains at least another element of $T^{\prime}$. By Theorem 3, the degree of every element $T^{\prime} \in Y$ is at least one and thus the sum of the degrees of all elements in $X$ is at least

$$
|Y|=\left(\begin{array}{l}
n \\
5
\end{array}\right) .
$$

Then there is a vertex in $X$ with degree at least

$$
\frac{|X|}{|Y|}=\frac{\left(\begin{array}{l}
n \\
5
\end{array}\right)}{\left(\begin{array}{l}
n \\
2
\end{array}\right)}=\frac{(n-2)(n-3)(N-4)}{60}
$$

In other words, there exists a pair $T$ of $\Phi$ such that any set $S^{\prime}$ homothetic to $S$ that contains them, contains at least one other element of $((n-2)(n-3)(n-4)) / 60$ five-subsets of $\Phi$. Allowing for redundancies (each $T$, together with any other element, belongs to $((n-3)(n-4)) / 2$ different sets), we obtain that any $S^{\prime}$ homothetic to $S$ containing both elements of $T$ contains at least $\lceil(n-2) / 301$ elements of $\Phi$.

\section{Proof of Theorem 3}

In this section we prove Theorem 3 . Some definitions and terminology are needed.

\subsection{Closest Covered Set Voronoi Diagram}

Let $S$ be a strictly convex compact set, such that $S$ contains the origin $(0,0)$ in its interior. Let $\Psi=\{\lambda S=\{\lambda x: x \in S\}: \lambda \geq 0\}$. All elements $\lambda S \in \Psi$ are homothetic to $S$. Call $(0,0)$ the vortex of $\Psi$. In turn we call $(0,0)$ the vortex of $\lambda S \in \Psi$.

Let $S^{\prime}$ be homothetic to $S$. Then $S^{\prime}$ is a translation by a vector $t=(a, b)$ of some $\lambda S \in \Psi$, that is, $S^{\prime}=t+\lambda S$. The vortex of $S^{\prime}$ is now defined to be the image of $(0,0)$ under this translation, i.e., vortex $\left(S^{\prime}\right)=t$.

Given any point $p$ on the plane and a convex set $S_{i}$, define the distance $d_{S}\left(p, S_{l}\right)$ to be the smallest $\lambda$ such that $S^{\prime}=p+\lambda S$ and $S^{\prime}$ contains $S_{i}$. Given two noncrossing sets $S_{i}$ and $S_{j}$, we may now define the vortex bisector $b_{S}\left(S_{i}, S_{j}\right)$ to be the set of points $p$ satisfying $d_{S}\left(p, S_{i}\right)=d_{S}\left(p, S_{j}\right)$.

It is easy to see that under the restrictions imposed on $S$, the vortex bisector of $S_{l}$ and $S_{j}$ is well defined and is always a simple curve that partitions $\mathfrak{R}^{2}-b_{S}\left(S_{i}, S_{J}\right)$ into two disjoint sets, one consisting of all points closer to $S_{i}$ than to $S_{j}$ and the other containing those closer to $S_{j}$ than to $S_{i}$.

At this point it is worth noting that if the noncrossing condition on $S_{i}$ and $S_{j}$ is dropped, then the vortex bisector $b_{S}\left(S_{i}, S_{j}\right)$ of $S_{i}$ and $S_{j}$ is not necessarily a simple curve. For 

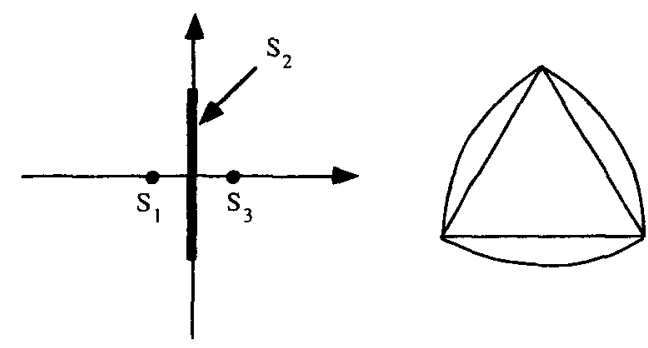

Fig. 2

instance, the reader may easily verify that if $S_{i}$ and $S_{j}$ are two line segments of the same length that cross at their midpoint, and $S$ a circle, then $b_{S}\left(S_{i}, S_{j}\right)$ consists of two lines that meet at the intersection point of $S_{l}$ and $S_{l}$.

Consider now a family $\Phi=\left\{S_{1}, \ldots, S_{n}\right\}$ of noncrossing compact convex sets. We may associate to each member of $S_{l} \in \Phi$ a Voronoi region $\operatorname{Vor}_{S}\left(S_{i}\right)$ consisting of all points $p$ on the plane such that $d_{S}\left(p, S_{l}\right) \leq d_{S}\left(p, S_{j}\right)$ for every $S_{j} \in \Phi, j \neq i$.

The set of regions $\left\{\operatorname{Vor}_{S}\left(S_{i}\right): i=1, \ldots, n\right\}$ thus obtained is called the closest covered set Voronoi diagram of $\Phi$ with respect to $S$ and is denoted by $\operatorname{Vor}(S, \Phi)$.

It is easy to verify that if the sites of $\Phi$ are points, then we obtain precisely the well-known Voronoi diagram under a convex function; see [3] and [8].

There are, however, some important differences between $\operatorname{Vor}(S, \Phi)$ and regular Voronoi diagrams. For example, for some elements of $\Phi$, we may have $\operatorname{Vor}_{S}\left(S_{i}\right)=\varnothing$. If

$$
\Phi=\left\{S_{1}=\{(-1,0)\}, S_{2}=\{(x, y): x=0,-2 \leq y \leq 2\}, S_{3}=\{(1,0)\}\right\}
$$

and $C$ is a disk with center at the origin, then $\operatorname{Vor}_{C}\left(S_{2}\right)=\varnothing$. See Fig. 2. Another example is obtained by letting $\Phi=\left\{S_{1}, S_{2}\right\}$, with $S_{1}$ and $S_{2}$ as defined above, and letting $S$ be a Reuleaux triangle $R$ such that the equilateral triangle used to generate it has its base parallel to the $x$-axis. Notice that in this case, any homothet to $R$ that contains $S_{2}$ contains $S_{1}$ in its interior, and thus $\operatorname{Vor}_{R}\left(S_{2}\right)=\varnothing$.

Given two convex sets $P$ and $Q$ such that $P \subset Q$, we say that $P$ splits $Q$ if $Q-P$ is not connected; see Fig. 3.

We now proceed to characterize those sets $S_{\imath} \in \Phi$ for which $\operatorname{Vor}_{S}\left(S_{t}\right)=\varnothing$. The following observation will be useful:

Observation 1. Let $S^{\prime}$ and $S^{\prime \prime}$ be two different strictly convex and homothetic sets. Then their boundaries intersect in at most two points.

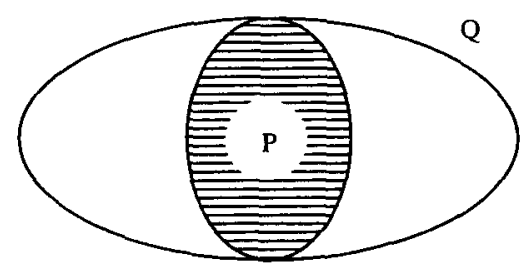

Fig. 3 


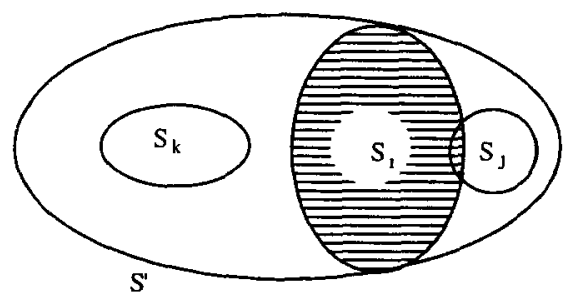

Fig. 4

Lemma 1. $\operatorname{Vor}_{S}\left(S_{i}\right)=\varnothing$ iff (a) $\operatorname{or}(\mathbf{b})$.

(a) There is an element $S_{j}$ of $\Phi=\left\{S_{1}, \ldots, S_{n}\right\}, i \neq j$, such that any $S^{\prime}$ homothetic to $S$ that contains $S_{l}$ contains $S_{J}$.

(b) There is a set $S^{\prime}$ homothetic to $S$ such that

(i) $S_{i}$ splits $S^{\prime}$ and

(ii) there are two different components $A, B$ of $S^{\prime}-S_{i}$ and two elements $S_{j}, S_{k}$ of $\Phi$ such that $S_{j} \subset A \cup S_{i}$ and $S_{k} \subset B \cup S_{i}$. See Fig. 4.

Proof. Suppose that $\operatorname{Vor}_{s}\left(S_{i}\right)=\varnothing$. If there is an $S_{j}$ satisfying (a) there is nothing to prove. Suppose then that for every $S_{j} \neq S_{i}$ there is an set $S_{i, j}$ homothetic to $S$ that contains $S_{i}$ and does not contain $S_{j}$. Among all sets homothetic to $S$ containing $S_{i}$, choose the one with the smallest area; call it $S^{\prime \prime}$. It is easy to show that due to the conditions imposed on $S, S_{i}$ splits $S^{\prime \prime}$. Since $\operatorname{Vor}_{S}\left(S_{i}\right)=\varnothing$ the vortex $v^{\prime \prime}$ of $S^{\prime \prime}$ belongs to $\operatorname{Vor}_{S}\left(S_{j}\right)$ for some $j \neq i$. This implies that $S_{j}$ is contained in $S^{\prime \prime}$. Since $S_{\imath}$ splits $S^{\prime \prime}$ and $S_{i}$ and $S_{j}$ do not cross each other, there is a component, say $A$, of $S^{\prime \prime}-S_{i}$ such that $S_{j}$ is contained in $A \cup S_{i}$. Assume that no other element of $\Phi$ is contained in $A \cup S_{i}$ (this restriction can be taken care of easily). Using the existence of $S_{i, j}$, and continuity arguments, it is easy to show that there is a set $S^{\prime}$ homothetic to $S$ that satisfies the following conditions:

(a) $S^{\prime}$ contains $S_{i}$ and $S_{j}$.

(b) $S_{1}$ splits $S^{\prime}$.

(c) $S_{j}$ intersects the boundary of $S^{\prime}$.

It is now easy to see by continuity that if all elements $S_{k}$ with $k \neq i, j$ are not contained in $S^{\prime}$, then we can find a homothetic copy $S^{\prime \prime}$ of $S$ that contains only $S_{i}$. (See Fig. 5). That is, the vortex of $S^{\prime \prime}$ belongs to $\operatorname{Vor}_{S}\left(S_{i}\right)$, which is a contradiction.

Conversely if condition (a) of our lemma is satisfied, then $\operatorname{Vor}\left(S_{i}\right)=\varnothing$. Suppose then that there is a set $S^{\prime}$ satisfying (b). Then it is easy to see using Observation 1 that any homothetic copy $S^{\prime \prime}$ of $S$ containing $S_{i}$ contains either $S_{j}$ or $S_{k}$ in its interior. In either case, the vortex of $S^{\prime \prime}$ belongs to $\operatorname{Vor}_{S}\left(S_{i}\right)$ or $\operatorname{Vor}_{S}\left(S_{J}\right)$, respectively, and thus $\operatorname{Vor}_{S}\left(S_{i}\right)=\varnothing$.

We also need the following result:

Lemma 2. If $\operatorname{Vor}_{S}\left(S_{\imath}\right) \neq \varnothing$, then it is connected. 


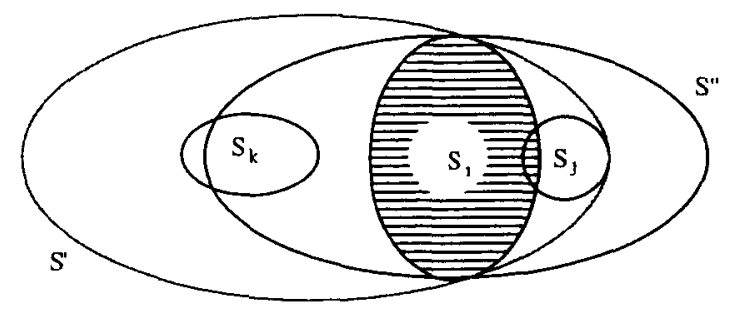

Fig. 5

The proof of this lemma is interesting on its own. To avoid breaking the flow of our paper we postpone its proof until the end of this section.

Next we prove:

Lemma 3. Let $\Phi$ be a family with at least four convex sets, and let $S_{i} \in \Phi$ be such that $\operatorname{Vor}_{S}\left(S_{l}\right)=\varnothing$. Then there is $S_{j} \in \Phi, i \neq j$, such that any $S^{\prime}$ homothetic to $S$ containing $S_{l}$ and $S_{j}$ contains another element of $\Phi$.

Proof. Suppose that $\operatorname{Vor}_{S}\left(S_{i}\right)=\varnothing$. By Lemma 1, either there is an $S_{k}$ different from $S_{i}$ that is contained in every homothet to $S$ containing $S_{l}$, or there are two elements of $\Phi$, say $S_{1}$ and $S_{2}$ different from $S_{i}$, and a homothetic copy $S^{\prime}$ of $S$ such that $S_{1}$ splits $S^{\prime}$ and there are two different components $A$ and $B$ of $S^{\prime}-S_{i}$ such that $S_{1}$ and $S_{2}$ are contained in $A \cup S_{i}$ and $B \cup S_{l}$, respectively.

In the first case, any homothet to $S$ containing $S_{i}$ and any other element $S_{j}$ of $\Phi$ different from $S_{k}$ contains $S_{k}$, and our result follows.

For the second case, we proceed as follows: Since $\Phi$ has at least four elements, there is $S_{j} \in \Phi$ different from $S_{1}, S_{2}$, and $S_{i}$. We now prove that any $S^{\prime \prime}$ homothetic to $S$ containing $S_{i}$ and $S_{J}$ contains $S_{1}$ or $S_{2}$.

Call $\beta_{A}$ the section of the boundary of $S^{\prime}-S_{i}$ that also belongs to the boundary of $A$. Suppose then that there is a homothetic copy of $S^{\prime \prime}$ that contains $S_{i}$ and $S_{j}$ and does not contain $S_{1}$. Then $S^{\prime \prime}$ intersects $\beta_{A}$ in at least two points (see Fig. 4). It now follows that $B$, and hence $S_{2}$, is totally contained in $S^{\prime \prime}$. Similarly we can prove that if $S_{2}$ is not contained in $S^{\prime \prime}$, then $S_{1}$ is, and our lemma is proved.

We are now ready to prove Theorem 3 :

Proof of Theorem 3. Consider a family $\Phi=\left\{S_{1}, \ldots, S_{5}\right\}$ of five disjoint compact convex sets and a strictly convex compact set $S$. Consider the Voronoi diagram $\operatorname{Vor}(S, \Phi)$. By Lemma 3 we can assume that $\operatorname{Vor}_{S}\left(S_{i}\right) \neq \varnothing, i=1, \ldots, 5$. Construct a graph $G$ in which for every region of $\operatorname{Vor}(S, \Phi)$ there is a vertex in $G$. Two vertices of $G$ are adjacent if their corresponding Voronoi regions are adjacent. Then by planarity arguments there are two different values $i, j$ such that $\operatorname{Vor}_{S}\left(S_{i}\right)$ and $\operatorname{Vor}_{S}\left(S_{j}\right)$ are not adjacent, otherwise a planar embedding of the complete graph on five vertices would be obtained, which is impossible. It is now follows that any $S^{\prime}$ homothetic to $S$ containing both $S_{i}$ and $S_{j}$ contains at least another element $S_{k}$ of $\Phi$, that is the set $S_{k}$ containing the vortex of $S^{\prime}$. 


\subsection{The Proof of Lemma 2}

We now prove that if a set $S_{i}$ is such that $\operatorname{Vor}_{S}\left(S_{i}\right) \neq \varnothing$, then $\operatorname{Vor}_{S}\left(S_{i}\right)$ is connected. We actually prove that if the interior $\operatorname{Int}\left(\operatorname{Vor}_{S}\left(S_{i}\right)\right)$ of $\operatorname{Vor}_{S}\left(S_{l}\right)$ is nonempty, then it is connected. This suffices to prove our result. To this end, we prove that for any two points $a^{\prime}$ and $a^{\prime \prime}$ in $\operatorname{Int}\left(\operatorname{Vor}_{S}\left(S_{i}\right)\right)$ there is a curve joining them totally contained in the interior of $\operatorname{Vor}_{S}\left(S_{i}\right)$.

We tackle first the case when $S_{l}$ is a convex polygon. Assume then that $S_{i}$ is a convex polygon, and let $a^{\prime}$ and $a^{\prime \prime}$ be points in the interior of $\operatorname{Vor}_{S}\left(S_{l}\right)$. Let $S\left(a^{\prime}\right)$ and $S\left(a^{\prime \prime}\right)$ be homothets to $S$ with vortices $a^{\prime}$ and $a^{\prime \prime}$, respectively, such that the boundaries $\partial\left(S\left(a^{\prime}\right)\right)$ and $\partial\left(S\left(a^{\prime \prime}\right)\right)$ of $S\left(a^{\prime}\right)$ and $S\left(a^{\prime \prime}\right)$ intersect the boundary $\partial\left(S_{l}\right)$ of $S_{i}$. Since $a^{\prime}$ and $a^{\prime \prime}$ belong to the interior of $\operatorname{Vor}_{S}\left(S_{i}\right), S\left(a^{\prime}\right)$ and $S\left(a^{\prime \prime}\right)$ do not contain any other elements $S_{j} \in \Phi$. Moreover, since we are assuming that $S_{i}$ is a convex polygon, $\partial\left(S_{l}\right)$ intersects $\partial\left(S\left(a^{\prime}\right)\right)$ and $\partial\left(S\left(a^{\prime \prime}\right)\right)$ at vertices of $S_{i}$.

We first reduce the case where $\partial\left(S\left(a^{\prime}\right)\right)$ and $\partial\left(S_{l}\right)$ intersect in exactly one vertex of $S_{i}$ to the case when $\partial\left(S\left(a^{\prime}\right)\right)$ and $\partial\left(S_{i}\right)$ intersect in at least two vertices of $S_{i}$. Suppose then that $\partial\left(S\left(a^{\prime}\right)\right)$ contains exactly one vertex, say $p$, of $S_{i}$. Let $L$ be the line segment joining $a^{\prime}$ to $p$. For every point $x$ of $L$ let $S(x, p)$ be the homothetic copy of $S$ with vortex $x$ and containing $p$ on its boundary. Clearly, $S(x, p)$ is contained in $S\left(a^{\prime}\right)$. If $x$ is sufficiently close to $p, S(x, p)$ does not contain $S_{i}$, and since $S\left(a^{\prime}\right)$ contains $S_{i}$ there is a point $y$ in $L$ such that $S(y, p)$ contains $S_{i}$ and intersects the boundary of $S_{i}$ at $p$ and another vertex of $S_{i}$ different from $p$. Similarly, we can assume that $\partial\left(S\left(a^{\prime \prime}\right)\right)$ intersects $\partial\left(S_{i}\right)$ in at least two vertices of $S_{i}$.

Let $p$ and $q$ denote the vertices of $S_{i}$ on the boundary of $S\left(a^{\prime}\right)$. Assume without loss of generality that the line $L$ through $p$ and $q$ is horizontal.

By assumption, $a^{\prime}$ belongs to the bisector $b_{S}(p, q)$ of $p$ and $q$ which is $y$-monotone since $p$ and $q$ have the same $y$-coordinate. This follows from a result in [8]. By definition, for each $a$ in $b_{S}(p, q)$ there is a unique homothetic copy $S(a)$ of $S$ with vortex $a$ whose boundary contains $p$ and $q$. Let $S^{+}(a)$ and $S^{-}(a)$ be the parts of $S(a)$ above (resp. below) the horizontal line $L$ (see Fig. 6).

Lemma 4. As a point a moves along $b_{S}(p, q)$ in the upward direction, $S^{+}(a)$ is strictly growing while $S^{-}(a)$ is strictly shrinking.

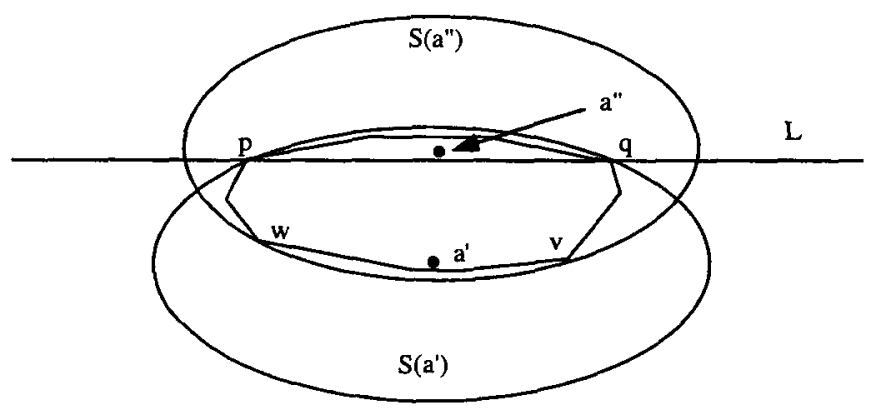

Fig. 6 


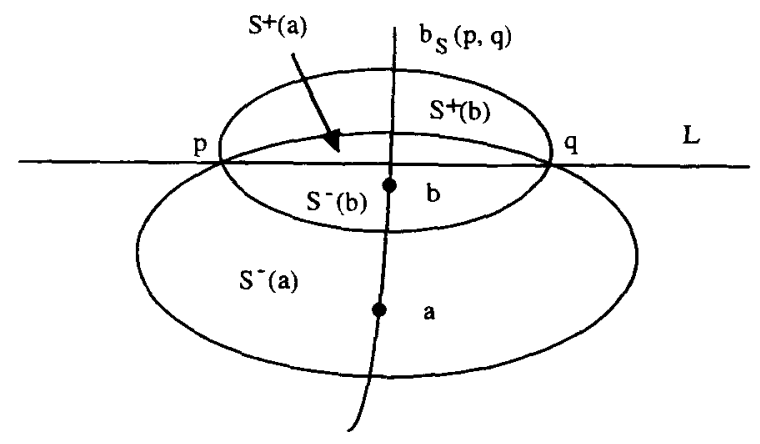

Fig. 7

Proof. Let $a$ and $b$ be on $b_{S}(p, q)$. Suppose without loss of generality that $S^{+}(b)$ contains $S^{+}(a)$. We have to show that, in this case, $a$ lies below $b$. Let $c$ be the intersection point of the common "outer" tangents of $S(a)$ and $S(b)$.

Clearly, $c$ is the center of a homotheticity between $S(a)$ and $S(b)$. First, assume that $c$ lies above $L$. From the point of view of $c$, the tangents intersect first $S(b)$ and then $S(a)$. Consequently, the ray from $c$ through $a$ and $b$ hits $b$ first. This implies that $b$ has a higher coordinate than $a$. A similar argument applies if $c$ is below $L$.

Now suppose that $S\left(a^{\prime}\right)$ and $S\left(a^{\prime \prime}\right)$ are such that $S^{+}\left(a^{\prime \prime}\right)$ contains $S^{+}\left(a^{\prime}\right)$ (see Fig. 7). In order to transform $S\left(a^{\prime}\right)$ into $S\left(a^{\prime \prime}\right)$, starting at $a^{\prime}$ we move a point $a$ upward along $b_{S}(p, q)$, and study how the set $S(a)$ with vortex at $a$ and whose boundary passes through $p$ and $q$ changes. By Lemma $4, S^{+}(a)$ grows while $S^{-}(a)$ shrinks.

Lemma 5. If $S(a)$ contains $S_{i}$, then the only set of $\Phi$ contained in $S(a)$ is $S_{i}$.

Proof. Suppose that some $S_{j} \neq S_{i}$ is also contained in $S(a)$. Since $S_{i}$ and $S_{j}$ do not cross, $S_{j}$ must be contained either in $S^{-}(a) \cup S_{i}$ which is contained in $S\left(a^{\prime}\right)$, or $S$, is contained in $S^{+}(a) \cup S_{l}$ which is contained in $S\left(a^{\prime \prime}\right)$, contradicting our assumptions.

We can now prove:

Lemma 6. Let $S_{i}$ be a convexpolygon such that $\operatorname{Int}\left(\operatorname{Vor}_{S}\left(S_{\imath}\right)\right) \neq \varnothing$. Then $\operatorname{Int}\left(\operatorname{Vor}_{S}\left(S_{i}\right)\right)$ is connected.

Proof. Let $a^{\prime}$ and $a^{\prime \prime}$ be points in the interior of $\operatorname{Vor}_{S}\left(S_{i}\right)$ with $S\left(a^{\prime}\right)$ and $S\left(a^{\prime \prime}\right)$ as defined before. We may assume that each of the boundaries of $S\left(a^{\prime}\right)$ and $S\left(a^{\prime \prime}\right)$ contains two vertices of $S_{i}$. Suppose that $S\left(a^{\prime}\right)$ intersects $S_{i}$ at $p$ and $q$ and $S\left(a^{\prime \prime}\right)$ intersects $S_{i}$ at $v$ and $w$. We have to prove that there is a path from $a^{\prime}$ to $a^{\prime \prime}$ entirely contained in $\operatorname{Int}\left(\operatorname{Vor}_{S}\left(S_{i}\right)\right)$, i.e., each point $a$ on this path is the vortex of a homothetic copy $S(a)$ of $S$ that contains $S_{i}$ and no other set $S_{j} \neq S_{i}$. By assumption, $S\left(a^{\prime}\right)$ and $S\left(a^{\prime \prime}\right)$ satisfy this condition. 


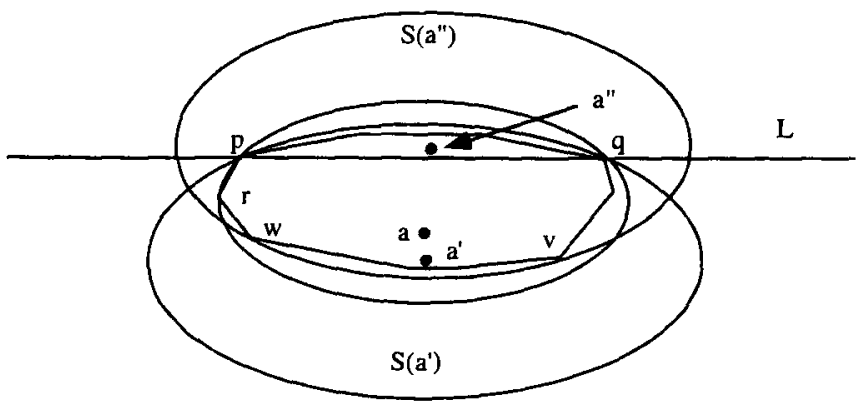

Fig. 8

There are two possible events that can prevent us from moving $a$ upward along $b_{S}(p, q)$. First, it could happen that the shrinking boundary of $S(a)$ hits a third vertex, say $r$ of $S_{i}$ (see Fig. 8). Note that it cannot happen that $r$ is a vertex on the boundary section of $S_{i}$ from $v$ to $w$ that does not contain $p$ and $q$; this would cause $S(a)$ to intersect $S\left(a^{\prime \prime}\right)$ in at least four points, which is impossible since $S\left(a^{\prime}\right)$ and $S\left(a^{\prime \prime}\right)$ are homothetic. At this point, we "update" $a$ ' to be $a$.

Now the vertices of $S_{i}$ contained on the part of $\partial\left(S\left(a^{\prime}\right)\right)$ contained in $S\left(a^{\prime \prime}\right)$ are not $p$ and $q$, they are now $r$ and $q$. Now we continue moving $a$ along the bisector $b_{S}(r, q)$ in the same way as before and update $a^{\prime}$ as we move along. Eventually, the boundary of $S^{+}(a)$ will hit the boundary of $S^{+}\left(a^{\prime \prime}\right)$. At this point, $S(a)=S\left(a^{\prime \prime}\right)$, and our result follows.

We are now ready to prove Lemma 2 .

Proof of Lemma 2. We prove now that $\operatorname{Int}\left(\operatorname{Vor}_{S}\left(S_{l}\right)\right)$ is connected. By Lemma 6 we can assume that $S_{i}$ is not a convex polygon. Let $a^{\prime}$ and $a^{\prime \prime}$ be points in the interior of $\operatorname{Vor}_{S}\left(S_{i}\right)$, and let $S\left(a^{\prime}\right)$ and $S\left(a^{\prime \prime}\right)$ be homothets to $S$ with vortices $a^{\prime}$ and $a^{\prime \prime}$ such that $S_{i}$ is contained in the interior of $S\left(a^{\prime}\right)$ and $S\left(a^{\prime \prime}\right)$. Let $S_{1}^{\prime}$ be a convex polygon containing $S_{i}$ such that $S_{i}^{\prime}$ is contained in $S\left(a^{\prime}\right) \cap S\left(a^{\prime \prime}\right)$. Since $S_{l}$ is a subset of $S_{t}^{\prime}$ then $\operatorname{Vor}\left(S_{i}^{\prime}\right)$ is contained in $\operatorname{Vor}\left(S_{i}\right)$. By Lemma 6 , there is a path from $a^{\prime}$ to $a^{\prime \prime}$ totally contained in $\operatorname{Int}\left(\operatorname{Vor}\left(S_{i}^{\prime}\right)\right)$ which in turn is contained in $\operatorname{Vor}_{S}\left(S_{i}\right)$. Our result now follows.

\section{Conclusions and Open Problems}

We have proved that for any family $\Phi$ of $n$ noncrossing compact convex sets, and $S$ strictly convex, there are two elements $S_{i}, S_{j} \in \Phi$ such that any $S^{\prime}$ homothetic to $S$ containing $S_{i}$ and $S_{j}$ contains at least $\lceil(n-2) / 30\rceil$ elements of $\Phi$. We believe that the bound stated in Theorem 2 is far from optimal. At this time we are unable to give a good estimate for an optimal solution to our problem, but we believe that a bound of about $n / 4$ or $n / 5$ is achievable.

For points and circles, it has been proved that if the elements of $P$ are vertices of 


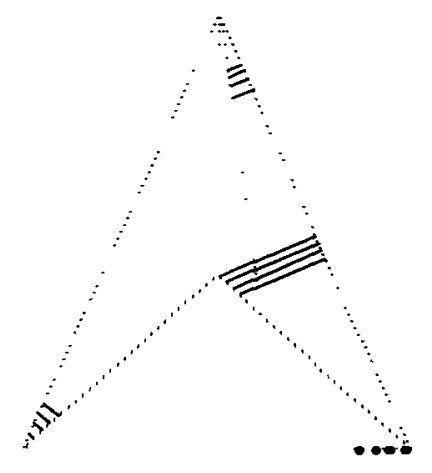

Fig. 9

a convex polygon, then there are two points of $P$ such that any circle containing them contains $\lceil(n-3) / 3\rceil$ points of $P$, and this is optimal [7]. This result does not extend, however, to the natural generalization to our problem. A family $\Phi$ of sets is called convexly independent if no element $S_{i}$ of $\Phi$ is contained in the convex closure of $\Phi-S_{i}$. We have an example consisting of $4 n$ elements such that for any pair of elements of $\Phi$ there is a circle containing them that contains at most $n$ elements of $\Phi$ (see Fig. 9).

In this more restricted case we venture the next conjecture:

Conjecture. Let $\Phi$ be a family of $n$ convexly independent sets, $S$ a convex set. Then there are two elements of $\Phi$ such that any $S^{\prime}$ homothetic to $S$ containing them contains at least $n / 4 \pm c$ elements of $\Phi, c$ a constant.

Finally, we mention that the construction of the new type of Voronoi diagrams introduced here can be carried out in $k O(n \log n)$ time, using techniques developed in [9], where $k$ is the time it takes to calculate the vortex bisector of two elements of $\Phi$.

\section{Acknowledgment}

The authors would like to thank the referees for their careful reading that helped to improve the presentation of this paper.

\section{References}

1. I. Bárány and D. G. Larman, A combinatorial property of points and ellipsoids, Discrete Comput. Geom. 5 (1990), 375-382.

2. I. Bárány, J. H. Schmerl, S. J. Sidney, and J. Urrutia, A combinatorial result about points and balls in Euclidean space, Discrete Comput. Geom. 4 (1989), 259-262.

3. P. Chew and S. Drysdale, Voronoi diagrams based on convex distance functions, Proc. 1 st ACM Symposium on Computational Geometry, Baltimore, MD, 1985, pp. 235-244.

4. H. Edelsbrunner, N. Hasan, R. Seidel, and X. J. Shen, Circles through two points that always enclose many points, Geom. Dedicata 32 (1989), 1-12. 
5. P. C. Fishburn, Interval orders and circle orders, Order 5 (1988), 225-234.

6. R. Hyward, A note on the circle containment problem, Discrete Comput. Geom. 4 (1989), 263-264.

7. R. Hyward, D. Rappaport, and R. Wenger, Some extremal results on circles containing points, Discrete Comput. Geom. 4 (1989), 253-258.

8. Ch. Ickling, R. Klein, M. Le, and L. Ma, Convex distance functions in 3-space are different, Proc. 9th ACM Symposium on Computational Geometry, 1993, pp. 116-123.

9. R. Klein, K. Melhorn, and S. Meiser, Randomized incremental construction of abstract Voronoi diagrams, Comput. Geom. Theory Appl. 3 (1993), 157-184.

10. V. Neumann-Lara and J. Umutia, A combinatorial result on points and circles in the plane, Discrete Math. 69 (1988), 173-178.

11. J. Urrutia, Partial orders and Euclidean geometry, in Algorithms and Order, ed. I. Rival, Kluwer, Boston, MA, 1989 , pp. 387-434.

Received December 7, 1994, and in revised form March 25, 1996. 